\title{
Digital Solutions for Cancer Survivorship Care
}

Ingrid Oakley-Girvan ${ }^{1}, \mathrm{PhD}, \mathrm{MPH} ;$ Sharon Watkins Davis ${ }^{2}, \mathrm{MPA}$; Michelle Longmire${ }^{2}$, MD

${ }^{1}$ Public Health Institute, Research, Oakland, CA, United States

${ }^{2}$ Medable, Inc, Palo Alto, CA, United States

Corresponding Author:

Ingrid Oakley-Girvan, $\mathrm{PhD}$, MPH

Public Health Institute

Research

555 12th Street, 10th Floor

Oakland, CA,

United States

Phone: 6562948

Fax: 2855501

Email: oakleygirvan@gmail.com

\section{Abstract}

Background: Both the National Cancer Institute (NCI) and Institute of Medicine have stressed the importance of survivorship care plans (SCP) for cancer patients/survivors and discussed the significance and importance of required input from survivors and advocates. However, there are many barriers to cancer care coordination and the creation of SCPs, including oncology staff time required to write them. Although survivors valued SCPs and liked them, few survivors or caregivers report receiving survivorship information and in some studies, reported no receipt of an SCP. Digital platforms can support cancer survivorship care by integrating with the existing Electronic Health Record and presenting information in a dynamic and user-friendly format that improves coordination and communication.

Objective: In this paper, we describe our involvement of stakeholders, including medical staff, patients/survivors and informal caregivers in developing a user-centered design for TOGETHERCARE, a smartphone app envisioned to provide critical functionality including planning and sharing of the SCP among survivors, physicians, and informal caregivers.

Methods: Two interviewers conducted a total of nine semi-structured interviews, including a convenience sample of three health care providers who work with cancer patients, three cancer patients/survivors, and three informal caregivers currently caring for cancer patients/survivors. The interviews with Spanish-speaking patients/survivors and caregivers were conducted with a translator. Notes from the interviews were transcribed into a prepared template. The results were compiled and coded by two members of the core team.

Results: We identified areas of consistency in responses between the three different groups in terms of how the application should work, as well as areas of difference. Additional suggestions for features for the application are also presented. Health care providers focused on the efficiency of using the application, features that would improve follow-up visits with patients and reduce the nursing triage, ER visits and readmissions. Survivors and caregivers were more focused on features that would provide assistance with patient appointment schedules, at-home medical tasks and activities of daily living. Although all three groups agreed that there is currently no systematic way for specialists to keep in touch with patients once they have moved to community care, and that SCPs would be useful, the practice of providing SCPs is rarely implemented. Survivors, caregivers, and providers all agreed that they have smartphones and that an app that includes the ability to communicate between the different groups, along with other features such as guidance on assisting with daily medical tasks and activities of daily living would be useful.

Conclusions: The pervasiveness of mobile devices and mobile app use provides an opportunity to make survivorship information and plans more readily available to caregivers and survivors, and to incorporate patient outcome reporting. Health care providers, cancer survivors, and informal caregivers all responded positively to a variety of features that could improve the efficiency of cancer care coordination and dynamic SCP provision.

(iproc 2018;4(2):e11717) doi: $10.2196 / 11717$

\section{KEYWORDS}

access to information; ambulatory care information systems; computer, handheld; medical records systems, computerized 


\section{Multimedia Appendix 1}

Full poster.

[PDF File (Adobe PDF File), 352KB-Multimedia Appendix 1]

Edited by T Hale; this is a non-peer-reviewed article. Submitted 27.07.18; accepted 29.08.18; published 17.09.18.

Please cite as:

Oakley-Girvan I, Davis SW, Longmire M

Digital Solutions for Cancer Survivorship Care

iproc 2018;4(2):e11717

URL: http://www.iproc.org/2018/2/e11717/

doi: $10.2196 / 11717$

PMID:

CIngrid Oakley-Girvan, Sharon Watkins Davis, Michelle Longmire. Originally published in Iproceedings (http://www.iproc.org), 17.09.2018. This is an open-access article distributed under the terms of the Creative Commons Attribution License (https://creativecommons.org/licenses/by/4.0/), which permits unrestricted use, distribution, and reproduction in any medium, provided the original work, first published in Iproceedings, is properly cited. The complete bibliographic information, a link to the original publication on http://www.iproc.org/, as well as this copyright and license information must be included. 